\title{
Identification of non-stationary dynamical systems using multivariate ARMA models
}

\author{
Mathieu Bertha*, Jean-Claude Golinval \\ University of Liège, Department of Aerospace and Mechanical Engineering, \\ Allée de la Découverte 9, 4000 Liège, Belgium
}

\begin{abstract}
This paper is concerned by the modal identification of time-varying mechanical systems. Based on previous works about autoregressive moving average models in vector form (ARMAV) for the modal identification of linear time invariant systems, and time-varying autoregressive moving average models (TV-ARMA) for the identification of nonstationary systems, a time-varying ARMAV (TV-ARMAV) model is presented for the multivariate identification of time-varying systems. It results in the identification of not only the timevarying poles of the system but also of its respective time-varying mode shapes. The method is applied on a time-varying structure composed of a beam on which a mass is moving.
\end{abstract}

Keywords: Time-varying systems, modal identification, vector auto-regressive moving average modeling, basis functions, moving mass problem

\section{Introduction}

In the field of structural engineering, modal analysis represents a significant part. Whether numerical or experimental, modal analysis is a mature topic when the assumptions of linearity and time-invariance are met for the structure to be analyzed. Nowadays, the challenges mainly focus on nonlinear modal analysis and time (or any other parameter) dependence of linear systems [1]. In both cases, the response signals recorded on the structure exhibit nonstationary behaviors.

The loss of the stationary assumption directly impacts the identification methods. New signal processing tools are then required to take the time variation into account. Time-frequency methods were designed for this purpose, the simplest one being the Short-Time Fourier Transform (STFT). This method simply applies the traditional Fourier transform on short time windows by assuming a piecewise stationarity of the signal. By applying this kind of methods, it is obvious that there is a trade-off between the frequency resolution and the ability to follow the variation of the signal properties. This kind of approach is also used for the modal analysis of time-varying structures by applying standard modal analysis methods on short sliding time windows where the response signals are assumed stationary. For example, the Stochastic Subspace Identification (SSI) method is applied on a bridge-like structure on short time windows in [2] and in [3] a variant of this method (the Crystal Clear SSI method) is applied on flight data of the Ariane 5 launcher to track the variation of its modal properties due to its decrease in mass with time.

Other time-frequency methods are also employed for their ability to track variations in the signals. One can cite the wavelet decomposition that can be used for the modal analysis of time-varying systems $[2,4,5,6]$. The tracking of instantaneous frequencies may also be performed by signal decomposition methods. The Hilbert-Huang Transform (HHT) [7] based on the Empirical Mode Decomposition (EMD) method and the Hilbert Vibration Decomposition (HVD) [8] method are able to decompose multicomponent signal into its

\footnotetext{
* Corresponding author

Email addresses: mathieu.bertha@ulg.ac.be (Mathieu Bertha), jc.golinval@ulg.ac.be (Jean-Claude Golinval)
} 
monocomponent constitutive parts, which are called Intrinsic Mode Functions (IMF). These two methods differ in the way they work but they are both based on the Hilbert transform and they are able to extract instantaneous amplitude and frequency of each monocomponent. In [9], the EMD method is used for the identification of a time-varying multiple degrees of freedom (MDOF) system and the HVD method is also applied on a MDOF system in [10, 11].

Another interesting way to proceed to the identification of time-varying systems is to build a parametric model in which the parameters to identify are free to vary with time. Such models, if they are properly designed, show good performances in terms of accuracy of the identification as well as with respect to their ability to track the dynamics variability of the underlying system. Autoregressive Moving-Average (ARMA) or State-Space (SS) models are often used for that purpose. In [12, 13, 14], the identification of time-varying systems is performed through the identification state-space models in which the state transition matrix is free to vary with time. An eigenvalue decomposition of this series of matrices results in what the author called the pseudo-modal parameters. In $[15,16]$ the estimation of time-varying ARMA models is performed by the Basis Function (BF) approach. In that method, the time-varying parameters of the model are expanded in a series of chosen time functions. This kind of models was first used in [17] for the estimation of evolutionary spectral density by a second-order Taylor series expansion of the parameters. In the field of structural dynamics, this method is applied in several different ARMA models (time dependent ARMA (TARMA), functional series TARMA (FS-TARMA), ...) by Fassois et al. [18, 19]. Most of the studies about time-varying systems deal with univariate time series and in the present paper, we use ARMA models in a multivariate form. Let us note that a similar study was performed in [20] but we focus here on the ability to well identify the varying mode shapes of the tested structure which are an important modal property in structural engineering. The latter mode shapes are validated by comparison with an analytical model of the problem. The identified mode shapes are then used for post identification of the position of the mass using an error localization process between a reference set of mode shapes and the time-varying ones.

The paper is organized as follows. Section 1 presents the method for the multivariate identification of TV systems. To illustrate the application of the method, an experimental demonstration structure was built and is firstly presented in Section 2. The time-varying identification is then performed and the obtained results are illustrated in Section 3. For validation purposes, a simple Rayleigh-Ritz model is built to accurately represent the dynamics of the problem and easily introduce the structural variation. It is presented in Section 4 together with its correlation with the experimental results. In Section 5, based on the identified mode shapes an application of localization of the variable part of the system is presented. The last section concludes the paper by recalling all the aspects of the work such as the difficulties that this kind of identification faces, the results we obtain and the possible applications of the identification of the mode time-varying shapes.

\section{Time-varying multivariate auto-regressive moving average model}

In this section, the method of Auto-Regressive Moving Average in Vector form (ARMAV) is first recalled in the field of linear time invariant (LTI) system identification. It leads to the determination of the modal properties in terms of poles (eigen frequencies $\omega_{r}$ and damping ratio's $\zeta_{r}$ ) as well as mode shapes $\boldsymbol{v}_{r}$ of the structure. Next, the method is extended to the time-varying behavior using the basis function approach as proposed in the FS-TARMA method but in a multivariate form. Accordingly, the parameters to be estimated become matrices instead of scalar coefficients.

\subsection{The ARMAV method for output-only modal identification}

The ARMAV method is able to perform modal identification of a structure based only on response measurements (output-only identification). A required assumption is that the external excitation generating the response of the structure is an uncorrelated white noise. This method is commonly used in the field of structural dynamics, see for example [21, 22, 23]. 
Let us note $\boldsymbol{y}[t]$ the $d \times 1$ multivariate responses measurement vector of the structure. The $\operatorname{ARMAV}(p, q)$ model of the output signal writes:

$$
\boldsymbol{y}[t]+\sum_{i=1}^{p} \boldsymbol{A}_{i} \boldsymbol{y}[t-i]=\boldsymbol{e}[t]+\sum_{j=1}^{q} \boldsymbol{B}_{j} \boldsymbol{e}[t-j]
$$

where the innovation $\boldsymbol{e}[t]$ is a zero-mean uncorrelated white noise process. The $p \boldsymbol{A}_{i}$ matrix coefficients represent the autoregressive part of the model that contains the dynamic information of the system. The other $q \boldsymbol{B}_{j}$ matrix coefficients constitute the moving average part of the model.

The relationship between an ARMAV model and a mechanical system is established in [24, 21]. Let us consider a mechanical system of order $n$ for which the governing equation writes

$$
\boldsymbol{M} \ddot{\boldsymbol{y}}(t)+\boldsymbol{C} \boldsymbol{y}(t)+\boldsymbol{K} \boldsymbol{y}(t)=\boldsymbol{f}(t)
$$

and suppose that we measure all the $n$ degrees of freedom. Transforming the system in its state-space representation, it may be shown that an $\operatorname{ARMAV}(2,1)$ is able to describe the dynamics of the system (2). Further, it is shown in [21] that in the presence of measurement noise, an $\operatorname{ARMAV}(2,2)$ should rather be used instead of $\operatorname{ARMAV}(2,1)$. But this result is valid if the dimension $d$ of the multivariate response vector matches exactly the actual dimension $n$ of the mechanical system. When it is not the case, $\operatorname{ARMAV}(2 m$, $2 m-1)$ or $\operatorname{ARMAV}(2 m, 2 m)$ are to be used with $m$ equal to the rounded up value of $n / d$.

\subsection{Time-varying ARMAV model}

Let us now consider the problem of time-varying mechanical systems where it is assumed that the rate of variation of the system is slow with respect to the period of vibration (i.e. the term $\dot{\boldsymbol{M}}(t) \dot{\boldsymbol{y}}(t)$ is negligible):

$$
\boldsymbol{M}(t) \ddot{\boldsymbol{y}}(t)+\boldsymbol{C}(t) \dot{\boldsymbol{y}}(t)+\boldsymbol{K}(t) \boldsymbol{y}(t)=\boldsymbol{f}(t)
$$

Because of the time dependence of the system matrices, the dynamic behavior of the system is also timedependent, i.e. its modal properties (eigen frequencies $\omega_{r}(t)$, damping ratio's $\zeta_{r}(t)$ and mode shapes $\boldsymbol{v}_{r}(t)$ ) are not constant anymore.

Regarding to the signal model (1), the time dependence have to be captured by the AR and MA coefficients. It follows that the ARMAV model in the framework of LTV systems is simply obtained by time-dependent matrices $\boldsymbol{A}_{i}[t]$ and $\boldsymbol{B}_{j}[t]$, i.e.:

$$
\boldsymbol{y}[t]+\sum_{i=1}^{p} \boldsymbol{A}_{i}[t] \boldsymbol{y}[t-i]=\boldsymbol{e}[t]+\sum_{j=1}^{q} \boldsymbol{B}_{j}[t] \boldsymbol{e}[t-j] .
$$

The coefficients to be estimated are now the time-varying $\boldsymbol{A}_{i}[t]$ and $\boldsymbol{B}_{j}[t]$ matrices. The key idea of the basis functions approach is to project these matrices on a previously selected set of known time functions $f_{k}[t]$.

$$
\begin{aligned}
\boldsymbol{A}_{i}[t] & =\sum_{k=1}^{r_{A}} \boldsymbol{A}_{i, k} f_{k}[t] \\
\boldsymbol{B}_{j}[t] & =\sum_{k=1}^{r_{B}} \boldsymbol{B}_{j, k} f_{k}[t]
\end{aligned}
$$

There are many possible choices for the basis functions and the most commonly used are polynomials (Legendre, Chebyshev, ...) or trigonometric functions, as in the following. Similar results may be obtained using each kind of basis but sometimes requiring different sizes of the function bases. Note that if the system exhibit a known structure in its varying properties, it could be used to provide structured basis functions to 
the method. In this way, the identification problem becomes a time invariant problem when looking for the projection coefficients $\boldsymbol{A}_{i, k}$ and $\boldsymbol{B}_{j, k}$. Introducing (5) and (6) into (4) yields to

$$
\boldsymbol{y}[t]+\sum_{i=1}^{p} \sum_{k=1}^{r_{A}} \boldsymbol{A}_{i, k} f_{k}[t] \boldsymbol{y}[t-i]=\boldsymbol{e}[t]+\sum_{j=1}^{q} \sum_{k=1}^{r_{B}} \boldsymbol{B}_{j, k} f_{k}[t] \boldsymbol{e}[t-j] .
$$

As previously said, the aim of the method is to identify the AR and MA projection coefficients. To do so, let us first gather all these coefficients in a single matrix $\Theta$ :

$$
\boldsymbol{\Theta}=\left[\boldsymbol{A}_{1,1}, \boldsymbol{A}_{1,2}, \cdots, \boldsymbol{A}_{1, r_{A}}, \boldsymbol{A}_{2,1}, \cdots, \boldsymbol{A}_{p, r_{A}}, \boldsymbol{B}_{1,1}, \cdots, \boldsymbol{B}_{q, r_{B}}\right] .
$$

In the same way, the product of the basis functions and the lagged values of the output and error term are also put in the following vector forms:

$$
\begin{aligned}
\boldsymbol{\phi}[t]^{T} & =\left[f_{1}[t] \boldsymbol{y}[t-1]^{T}, f_{2}[t] \boldsymbol{y}[t-1]^{T}, \cdots, f_{r_{a}}[t] \boldsymbol{y}[t-p]^{T}\right] \\
\boldsymbol{\psi}[t]^{T} & =\left[-f_{1}[t] \boldsymbol{e}[t-1]^{T},-f_{2}[t] \boldsymbol{e}[t-1]^{T}, \cdots,-f_{r_{b}}[t] \boldsymbol{e}[t-q]^{T}\right]
\end{aligned}
$$

The prediction error of the model can now be expressed by subtracting the estimate of the response $\hat{\boldsymbol{y}}[t, \boldsymbol{\Theta}]$ from the response signal itself $\boldsymbol{y}[t]$ :

$$
\hat{\boldsymbol{e}}[t, \boldsymbol{\Theta}]=\boldsymbol{y}[t]-\hat{\boldsymbol{y}}[t, \boldsymbol{\Theta}]
$$

where the estimate of the output signal is given by

$$
\hat{\boldsymbol{y}}[t, \boldsymbol{\Theta}]=-\sum_{i=1}^{p} \sum_{k=1}^{r_{A}} \boldsymbol{A}_{i, k} f_{k}[t] \boldsymbol{y}[t-i]+\sum_{j=1}^{q} \sum_{k=1}^{r_{B}} \boldsymbol{B}_{j, k} f_{k}[t] \boldsymbol{e}[t-j] .
$$

Using the notations (8), (9) and (10), the prediction error becomes

$$
\hat{\boldsymbol{e}}[t, \boldsymbol{\Theta}]=\boldsymbol{y}[t]+\boldsymbol{\Theta}\left[\begin{array}{l}
\boldsymbol{\phi}[t] \\
\boldsymbol{\psi}[t]
\end{array}\right]
$$

The matrix regression parameters of the system can be found by minimizing a positive scalar cost function of the modeling error with respect to the parameters. A commonly used cost function is the Sum of Squared Errors (SSE) defined as

$$
V(\boldsymbol{\Theta})=\frac{1}{N} \sum_{t=1}^{N} \hat{\boldsymbol{e}}[t, \boldsymbol{\Theta}]^{T} \hat{\boldsymbol{e}}[t, \boldsymbol{\Theta}],
$$

where $N$ is the number of data samples. A good estimate of $\boldsymbol{\Theta}$ is given by the minimum of $V(\boldsymbol{\Theta})$, i.e.

$$
\boldsymbol{\Theta}=\arg \min _{\boldsymbol{\Theta}} \frac{1}{N} \sum_{t=1}^{N} \hat{\boldsymbol{e}}[t, \boldsymbol{\Theta}]^{T} \hat{\boldsymbol{e}}[t, \boldsymbol{\Theta}] .
$$

The latter equation leads to a nonlinear optimization problem because $\boldsymbol{\psi}[t]$ depends on the error term which itself depends on the $\boldsymbol{\Theta}$ parameter. To solve this problem, the Multi Stage Least Squares (MSLS) method [25] is used here. It consists in building a sequence of linear least squares problems. This method applied in the scope of autoregressive moving average estimation is performed in two steps. First, a high-order autoregressive model is used to fit the data which requires only a least square estimate as the nonlinearity is located in the MA part. Once this model is known, it is used to get an estimate of the innovation $\hat{\boldsymbol{e}}\left[t, \boldsymbol{\Theta}_{\text {high order }}\right]$. In the second step, an ARMAV model is estimated by using the innovation as a known input. The process is then iterated by updating the output and the innovation. The drawback of the MSLS method compared to other nonlinear optimization scheme (such as Gauss-Newton or Levenberg-Marquardt) is that a decrease in the cost function is not ensured. The latter kind of optimization problems have good convergence properties but often suffer of high computation costs involved in the computation of the gradient and Hessian matrix of the cost function. Further, they also require a good initial guess for the model parameters to converge to the global optimum. 


\subsection{Computation of the modal parameters}

Once the basis functions coefficients are identified using the above method, the time-varying autoregressive matrix coefficients can be computed at any time instants using (5). These coefficients are representative of the instantaneous dynamics of the system at time $t$ as if it was fixed at this time $t$. In this so called frozen-time approach, the obtained parameters are a first approximation of those of the actual time-varying system and it is shown in $[26,27]$ that this approximation by the frozen-time approach converges to the true parameters as the variation rate between the system properties and its dynamics decreases.

The corresponding modal parameters at time $t$ are obtained through the so called companion matrix of the autoregressive part of the model:

$$
\boldsymbol{C}=\left[\begin{array}{ccccc}
-\boldsymbol{A}_{1}[t] & \boldsymbol{I} & \mathbf{0} & \cdots & \mathbf{0} \\
-\boldsymbol{A}_{2}[t] & \mathbf{0} & \boldsymbol{I} & \cdots & \mathbf{0} \\
\vdots & \vdots & \vdots & \ddots & \vdots \\
-\boldsymbol{A}_{p-1}[t] & \vdots & \vdots & & \boldsymbol{I} \\
-\boldsymbol{A}_{p}[t] & \mathbf{0} & \mathbf{0} & \cdots & \mathbf{0}
\end{array}\right]
$$

It can be shown that this matrix (or some variant of it) are related to the state-space representation of the model [25]. The eigenvalues $\mu_{r}$ of the companion matrix are the discrete-time poles of the system and are related to the poles of the system $\lambda_{r}$ by

$$
\mu_{r}=e^{\lambda_{r} \Delta t},
$$

where $\Delta t$ is the sampling time step of the recorded signals. The $d$ first components of the corresponding eigenvectors give the mode shapes of the system.

\section{Modal identification of the experimental system}

\subsection{Presentation of the test structure}

The system to be tested in this paper consist in an aluminum beam supported at its ends on which a mass is traveling as shown in Figure 1. It is a 2.1-meter long beam with a rectangular cross section of $8 \times 2$ centimeters. Both ends are supported by springs and bearing elements that let the rotation free at the supports levels. The mass is chosen to be sufficiently heavy to influence the dynamics of the system. The system is randomly excited by a shaker and its response is recorded by twelve accelerometers evenly distributed on the beam. This kind of excitation is used to always excite all the modes in the frequency range of interest but also because it is a usual assumption if we want to study a system using output-only measurements. The traveling mass is a $3.5-\mathrm{kg}$ steel block which is not negligible with respect to the mass of the beam $(\approx 9 \mathrm{~kg})$.

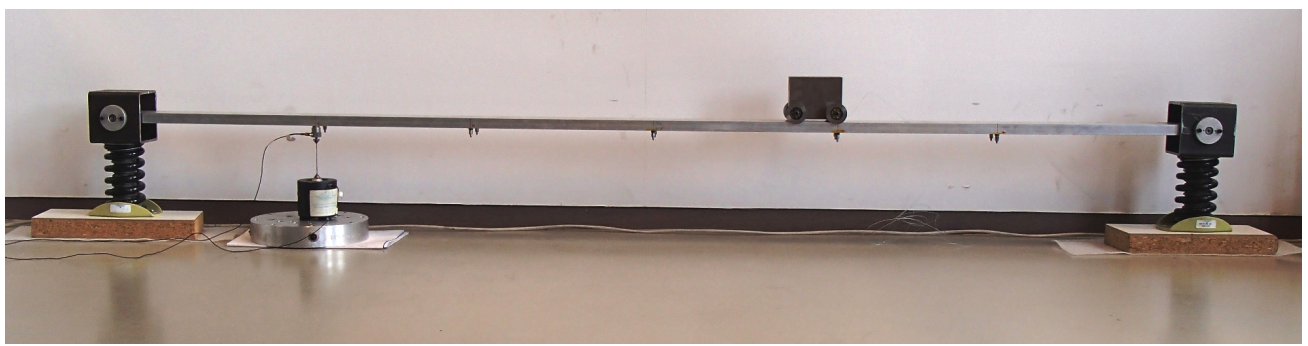

Figure 1: The supported beam on which the mass is moving.

This type of system is a recurrent structure used to test methods able to study time variant systems $[2,18,28]$. 


\subsection{LTI modal analysis of the beam subsystem}

A LTI modal analysis of the beam is first performed to serve as reference. Two sensors are placed at both ends in front of the springs and five pairs of sensors are located on each side of the beam in order to be able to detect bending as well as torsion modes. A schematic model of the measurement setup is shown in Figure 2. For all the tests, the system is randomly excited using a shaker at coordinate 3.

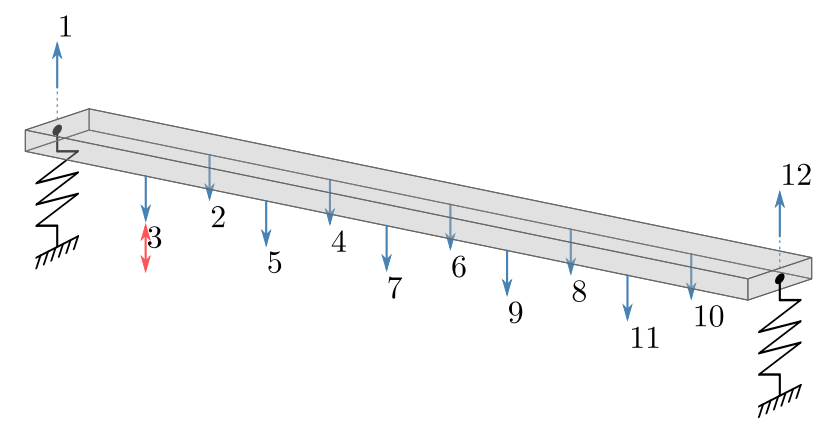

Figure 2: Scheme of the excitation and recording coordinates.

Data acquisition and excitation control are carried out using the LMS Scadas Mobile system. Signal processing and modal analysis itself are performed using the PolyMAX method [29] available in LMS Test.Lab. The results of the identification are summarized in Figure 3 and Table 1.

\begin{tabular}{|c|c|c|c|}
\hline Mode \# & $f_{r}[\mathrm{~Hz}]$ & $\zeta_{r}[\%]$ & Type of mode \\
\hline 1 & 99.86 & 0.32 & First bending \\
\hline 2 & 30.12 & 0.52 & $\begin{array}{l}\text { Opposite phase spring motion with } \\
\text { small second bending }\end{array}$ \\
\hline 3 & 38.6 & 0.65 & $\begin{array}{l}\text { In phase spring motion with small first } \\
\text { bending }\end{array}$ \\
\hline 4 & 53.14 & 0.27 & Second bending \\
\hline 5 & 62.17 & 1.56 & Rotation around the beam axis \\
\hline 6 & 99.70 & 0.28 & Third bending \\
\hline 7 & 168.60 & 0.99 & Fourth bending \\
\hline
\end{tabular}

Table 1: Experimental modal parameters of the supported beam.

\subsection{Time-Varying modal analysis of the beam-mass system}

For this test, the random excitation is turned on and the mass is pulled by hand using a simple wire while its displacement is recorded by a laser position sensor. The acquisition time is around 50 seconds but only the part when the mass is moving from the left to the right end is kept. The total amount of data is a record of 42 second sampled at $400 \mathrm{~Hz}$ for each channel. The output of the laser sensor corresponds to a linearly increasing voltage from $0 \mathrm{~V}$ (when the mass is located at the left end of the beam) to $10 \mathrm{~V}$ (when the mass reaches the right end). For illustration, Figure 4 shows the displacement of the mass with respect to time during the experiment. This motion has a strong influence on the dynamics of the system. When the mass travels along the beam, it locally increases the inertia force at its instantaneous position.

To show the effect of the additional mass on the system dynamics, time-frequency decomposition methods are useful. Figure 5 shows the evolution of the frequencies of the system with respect to time using the wavelet decomposition. The superimposed black dashed lines correspond to the frequencies that were identified in the previous LTI modal analysis of the alone beam subsystem (Table 1). First, it can be observed that the frequencies of the LTI subsystem act as upper bound for their equivalent in the time-varying case. This is not surprising as only an additional mass is introduced and the stiffness of the structure has not changed. 


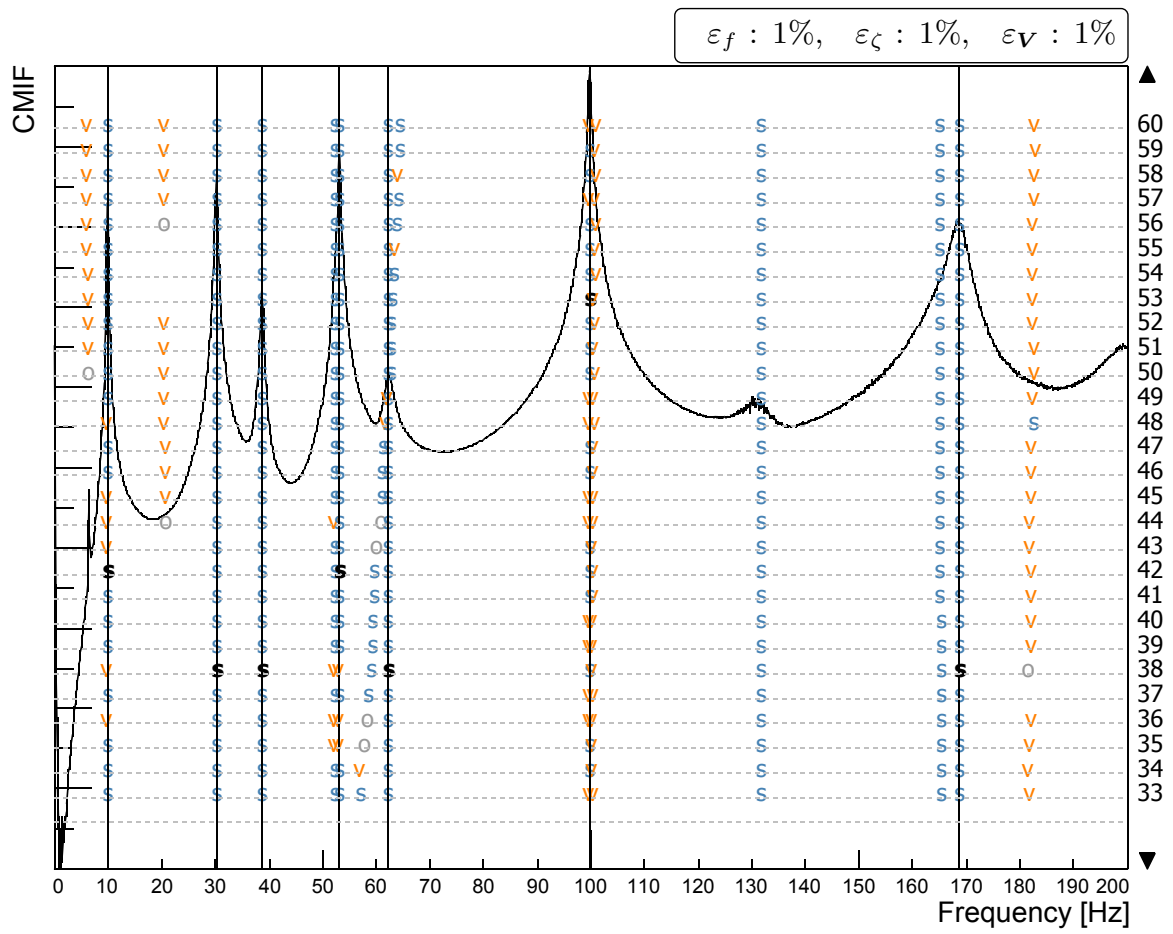

Figure 3: Stabilization diagram of the beam subsystem. The selected poles are appear as bold black s.

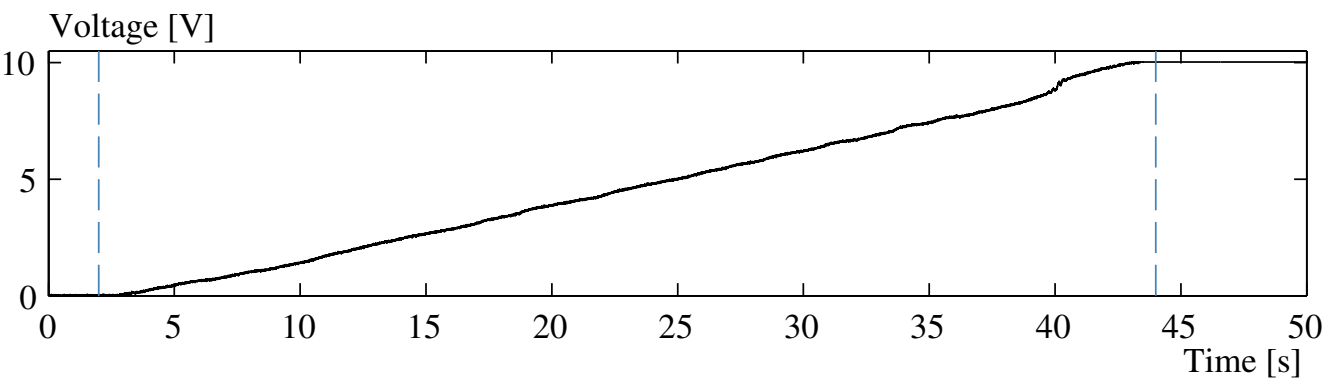

Figure 4: Displacement of the mass with respect to time. The dashed lines represent the selected portion of the data used in the following identification.

The instantaneous frequencies of the bending modes show a series of minima and maxima. It is easy to understand in this example that the maximum frequency drops occur when the mass is located at antinodes of vibration because the added inertia forces are maximum at these positions. Conversely, when the mass is located at a node of vibration of a specific mode, the participation of the mass to the response of that mode completely disappears and the frequency of the mode comes back to its initial value. Finally, the perturbation of the mass on the rotation vibration mode (initially at $62.17 \mathrm{~Hz}$ ) is more simple. Indeed, it just corresponds to a decrease in frequency with no great dependence with respect to the position of the mass. In this particular example, it happens that the frequency of the rotation mode becomes very close to the frequency of the fourth mode of vibration initially at $53.14 \mathrm{~Hz}$. Further, because the latter mode is a bending mode, it is more influenced by the position of the mass than the rotation mode. It appears now that these two modes show some crossings between their frequencies, what could make the identification difficult. 


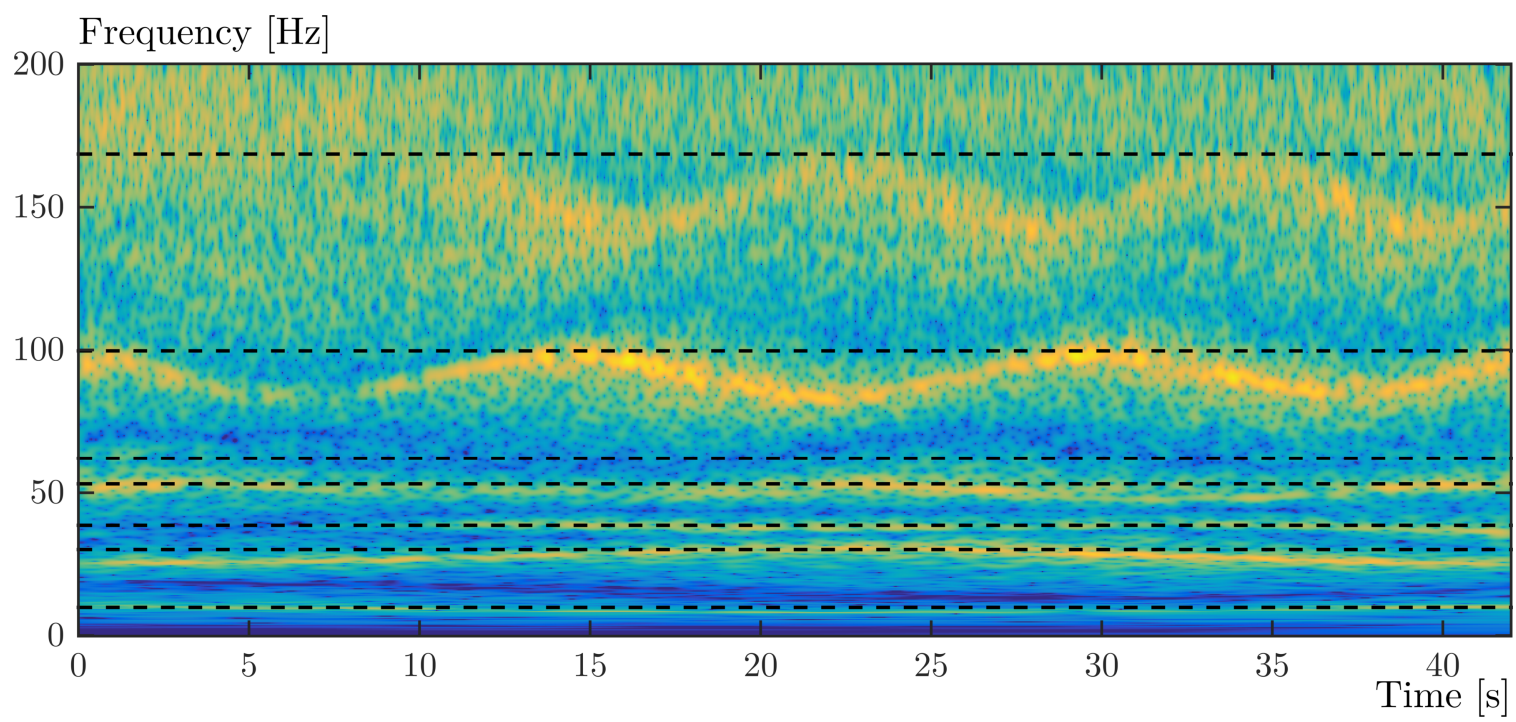

Figure 5: Wavelet decomposition of the response of sensor 3. The black dashed lines represent the natural frequencies of the system without the moving mass.

The time-varying identification method described earlier is now applied to identify the time-varying AR and MA matrices (5) and (6). The first step is to select the model to be applied to the data. The parameters to tune are the AR order, $p$, the MA order, $q$, the type of basis functions and their number $\left(r_{A}\right.$ and $r_{B}$, for the AR and MA parts, respectively). The model orders and the number of basis functions should be sufficient to properly fit the data but, according to the principle of parsimony, one should not introduce extra-parameters in the model if not necessary for the identification [30]. This also has an impact on the choice of the basis functions. Some bases may be more suitable than others depending on the system, especially if it has an a priori known behavior (such as periodically varying system for example). A smart choice of the type of the basis may require less functions for a similar fitting accuracy, which means less coefficients to identify. Usually in traditional modal analysis, a large number of models with increasing orders are used to identify a dynamic system and the results are plotted in a stabilization diagram such as the one shown in Figure 3. The selection of the poles is then performed manually or automatically using specific algorithms (e.g. clustering approach). A direct extension of the concept of stabilization diagram to the time-varying analysis would not be convenient because of the additional time dimension.

Several indicators exist to measure the fitting quality of the model taking its complexity into account. For example, one can cite among others the Akaike Final Prediction Error (FPE) or the Akaike Information Criterion (AIC) $[22,25]$. These two criteria are given by

$$
\begin{aligned}
F P E & =V(\boldsymbol{\Theta}) \frac{1+\frac{\delta}{N}}{1-\frac{\delta}{N}}, \\
A I C & =N \log [V(\boldsymbol{\Theta})]+2 \delta,
\end{aligned}
$$

where $\delta$ is the number of parameters to be estimated and $N$ the number of time samples. In our case, the number of parameters $\delta$ is the number of all the coefficients in all the projection matrices in (8) so that

$$
\delta=d^{2} p r_{A}+d^{2} q r_{B},
$$

where $d$ is the dimension of the output response vector $\boldsymbol{y}[t]$.

Once the model orders are fixed, the difficulty is to deal with the number of calculated poles and mode shapes. Indeed, the vector ARMA model leads to a number of poles increasing linearly with both the model order $p$ of the AR part and the number of measurement degrees of freedom $d$ (the dimension of the companion 
matrix (16) is $p d \times p d$ ). The dimension of the companion matrix may grow rapidly and exceed the actual number of modes in the frequency range of interest. Because of that, a huge number of spurious poles are calculated besides the physical ones. A discrimination should then be performed in order to get clear results. In classical modal analysis this is done using the stabilization diagram. For time-varying systems, the idea followed here retain a number $M$ of modes that looks the more physical among all the modes. In [31] the subset selection containing physical modes is done based on the radial distribution of the poles in the complex plane and only the $M$ poles closest to the unit circle are retained at any time step. This kind of discrimination is based on the fact that the physical modes are lightly damped with respect to the spurious ones. This method can be easily implemented here but in our case we also have another information that can be exploited: the mode shapes. The mode shapes may also serve for discrimination purposes between physical and spurious modes. Indeed, the physical modes of a real mechanical structure usually appear well aligned in the complex plane conversely to the spurious ones that exhibit a large dispersion. One way to quantify the aligned or scattered behavior of a mode is to compute its mean phase (MP) and mean phase deviation (MPD) which can be seen as the variance of the phase from the mean phase. Obviously, the ideal value of the MPD for a physical mode is close to $0^{\circ}$. In the Identification process, we will then retain only the $M$ modes with the lowest time-averaged MPD. Note that if we do not know the actual number of physical modes, an automatic clustering process could be applied to distinguish the modes with a MPD close to zero from the others. In Figure 6, an example of a physical mode 6(a) and a spurious mode 6(b) are shown. The mean phase for each mode is drawn together with their mean phase deviation. It is clearly visible that the spurious modes exhibit large MPD with respect to physical ones.

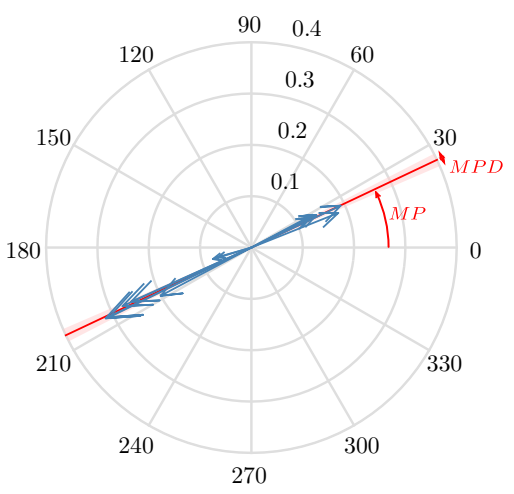

(a) Physical mode (low MPD)

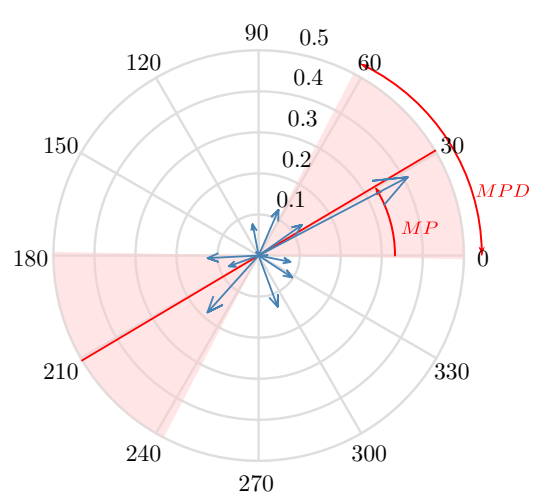

(b) Spurious mode (high MPD)

Figure 6: Illustration of the mode phase dispersion in the complex plane for the mode shapes at $t=15 s$. (a) is a physical mode at $28.65 \mathrm{~Hz}$ and (b) is classified as a spurious one at $185.14 \mathrm{~Hz}$.

\section{Results of the identification}

As stated above, in order to estimate the seven modes observed previously in the stationary analysis, the autoregressive order $p$ should be 2 . Indeed 7 modes are to be identified in the frequency band and 12 sensors are used. Using $p=2,12$ complex conjugated pairs of modes may be identified in which we would expect to be able to identify 7 physical and 5 spurious modes. The order being fixed, it remains now to select the size of the two function bases. To do so, a set of simulation were launched with $p=2$ and $q=2$ and varying sizes of the two sets of functions corresponding to the AR and MA parts. For this analysis, a Fourier basis is chosen which is composed of a unit function and a set of pairs of sine and cosine functions. The sizes of the two bases of functions are selected using the FPE and AIC criteria. The set of model parameters that minimize those two criteria in the case $p=q=2$ are $r_{A}=9$ and $r_{B}=3$ basis functions for the AR and MA parts, respectively. Even if the latter couple of sizes for the functions bases gives the minimum values 
for both the FPE and AIC criteria, few other sets of parameters give similar FPE and AIC values and are also considered. We observed that the $\operatorname{ARMAV}(2,2)$ model with $r_{A}=11$ and $r_{B}=1$ is located close to the minimum of the two criteria but gives slightly better results for the same number of parameters to identify. Figure 7 shows these two results keeping only the 7 modes that are the most physical with respect to the MPD criteria.

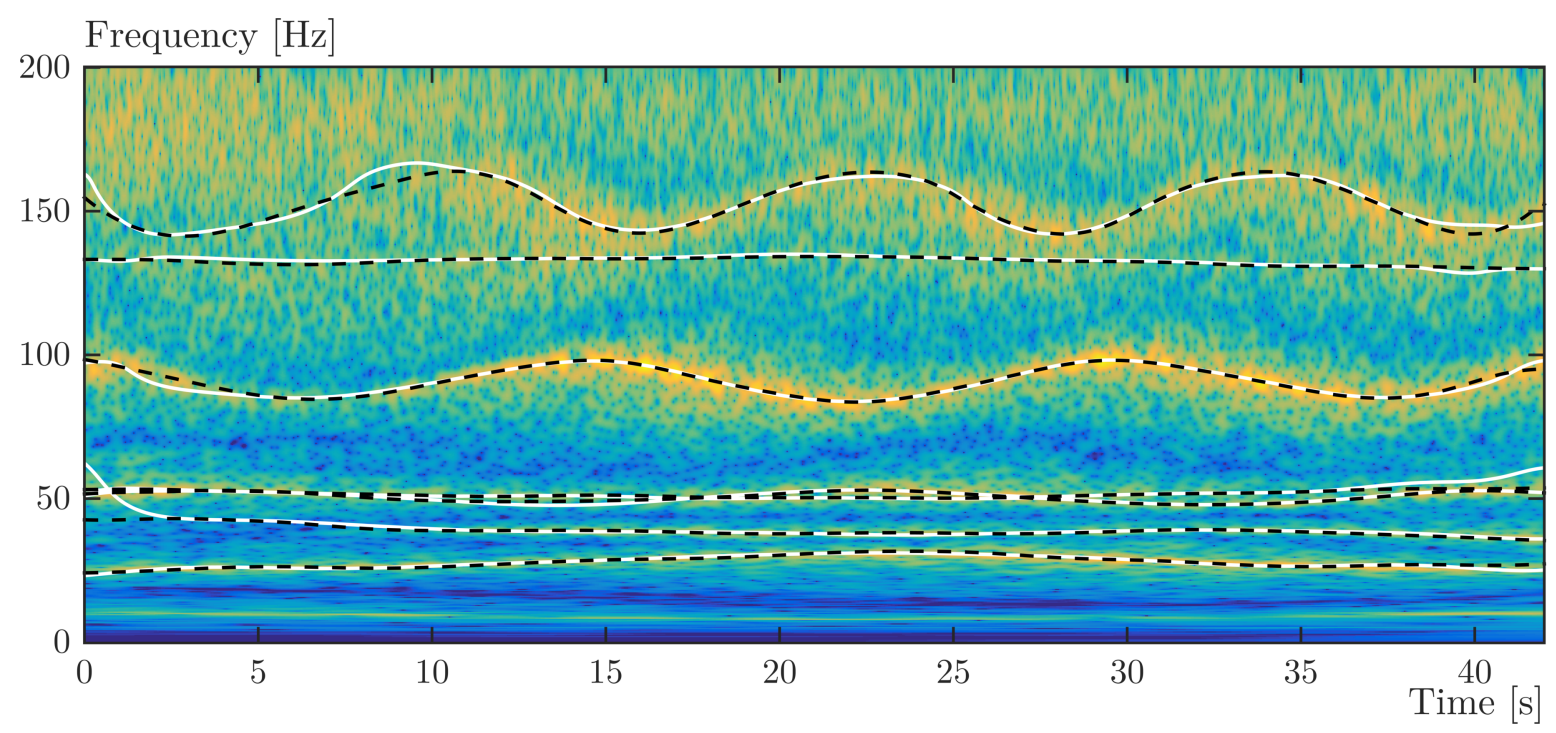

Figure 7: Results of the identification using TV-ARMAV $(2,2)$ models. White: $\operatorname{TV}-\operatorname{ARMAV}(2,2), r_{A}=9, r_{B}=3$; dashed black: TV-ARMAV $(2,2), r_{A}=11, r_{B}=1$.

At this stage, some comments may be drawn. Firstly, the first mode (with the lowest frequency) was not identified even if it is the less perturbed by the moving mass. Secondly, a constant mode close to 130 $\mathrm{Hz}$ is identified. Looking to the stabilization diagram of the initial structure (Figure 3 ) it corresponds to a low amplitude mode. Finally, the third mode (close to $40 \mathrm{~Hz}$ ) is not well identified during the 5 first seconds. Then, in order to better identify this latter mode and to identify the lowest frequency mode, other model structures are considered. Increasing the MA order $q$ to 3 , the model with $r_{A}=9$ and $r_{B}=1$ basis functions minimizes both the FPE and AIC criteria. This model gives a good estimate of the mode close to $40 \mathrm{~Hz}$. Finally, to identify the lowest frequency mode, the autoregressive order has to be increased up to $p=6$ to get a good result. Due to that order, the number of spurious poles considerably exceeds the number of physical poles (36 pairs of complex conjugated modes are calculated).

The final set of results is then obtained by selecting in the latter three models the modes that are well identified. The selection of the physical modes using the MPD criteria has another advantage here too. Once, for each model the physical modes are discriminated, it is possible to compare each occurrence of each mode in several models in order to keep the best one. We are then not restrained in a single model structure. Figure 8 illustrates the final set of identified time-varying modes. As we deal with multivariate models, the mode shapes corresponding to the identified frequencies are also determined as the eigenvectors of the companion matrix. As highlighted with the frequency results, the mode shapes are expected to be the most affected when the mass is located at antinodes of resonance. To show the effect of the mass on the mode shapes, let us take as example the sixth mode of vibration, exhibiting the third-bending mode of the beam. Because the mode is time-varying, only few samples corresponding to particular positions of the mass are selected for graphical representation as illustrated in Figure 9. To have a more complete view of the obtained results, an animation is provided together with the present article and the reader is encouraged to have a look at it. This video represents, hundred equally-spaced time spots, the results in terms of frequency and mode shapes for all the modes identified in the present experiment.

At first sight, it is easy to see in Figures 9(b) and 9(d), when the mass is located at two nodes of vibration, 


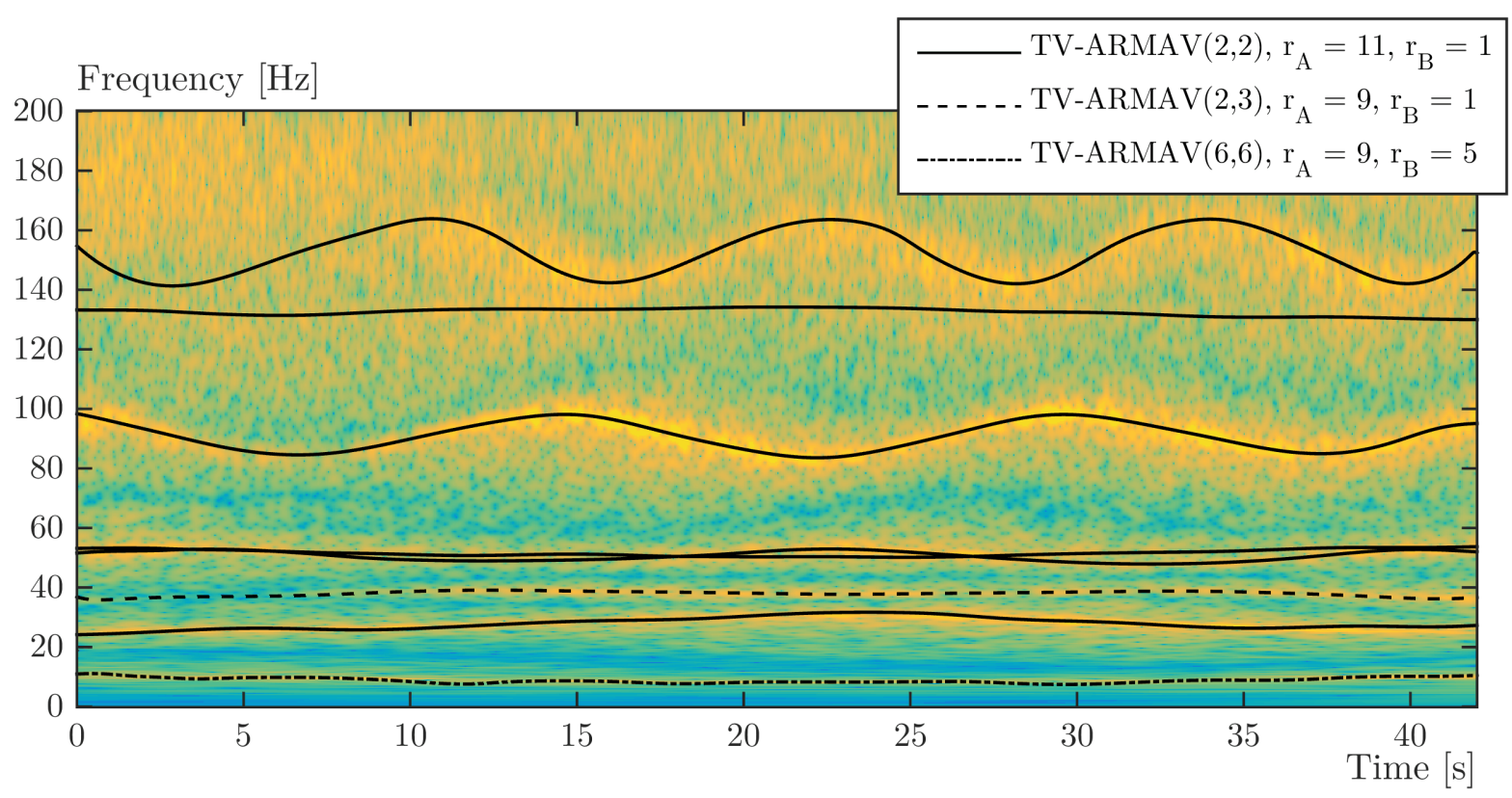

Figure 8: The final set of results is built by extracting modes from three different model structures.

that the mode shape is not perturbed by the presence of the mass. The antinodes have in that two cases the same magnitude. Now, taking a look to the three other figures $(9(\mathrm{a}), 9(\mathrm{c})$ and $9(\mathrm{e}))$ showing the deflection of the third bending mode when the mass is located at its anti nodes of vibration, the amplitude of the shape at the mass position decreases with respect to the magnitude of the two other antinodes. This is due to the fact that the mass has only a local effect.

\section{Comparison with a mathematical model}

In order to validate the identification results previously obtained, a mathematical model of the system is built using the Rayleigh-Ritz approach [32]. Further, once the beam subsystem is modeled, the moving mass is introduced as a punctual element at a varying coordinate about the $x$-axis of the beam.

The modeling is performed in two steps. First, A model of the beam on the two support springs is created, then updated using the results of the preliminary LTI analysis in Section 2.2 in order to overcome the lack of knowledge of some elements, mainly at the springs level. The moving mass is then introduced as a lumped mass/inertia in the system.

\subsection{Initial model and model updating}

The principle of the Rayleigh-Ritz approach is to build an approximate of the continuous solution using an approximation of the displacement field of the system in a basis of admissible trial function. The model of the system is shown in Figure 10. The beam of length $L$ and mass and inertia per unit length $m$ and $I$ has bending and torsion rigidities $E I_{y}$ and $G J$, respectively. The problem is solved for both bending and torsion modes separately. The method needs to identify all the contributions to the potential and kinetic energies involved in the system. Looking to the continuous beam, its vertical motion as well as the rotation of its cross section bring some kinetic energy to the system. Concerning its potential energy, it comes from its bending and torsion deformations. In addition to the motion of the beam, the supports will also influence the dynamics. They are modeled as stiffnesses in both vertical translation and rotation at the two extremities of the beam with values equal to $k_{t}$ and $k_{r}$, respectively. Further, an equivalent mass, $M_{0}$ is also added at both ends to take into account the supporting steel boxes and the equivalent mass of the springs. 


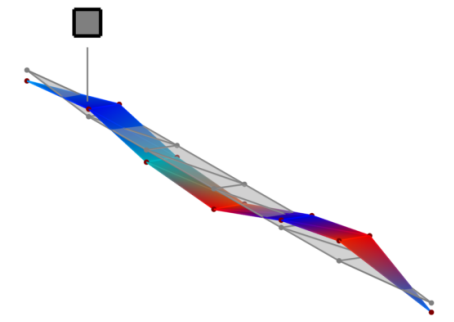

(a) Time: $t=6.7 \mathrm{~s}, f=84.55 \mathrm{~Hz}$

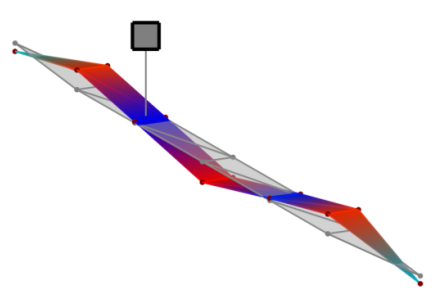

(b) Time : $t=14.7 \mathrm{~s}, f=98.13 \mathrm{~Hz}$

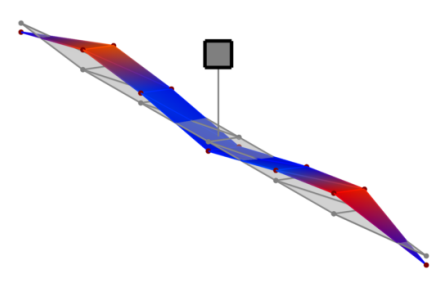

(c) Time : $t=22.2 \mathrm{~s}, f=83.58 \mathrm{~Hz}$

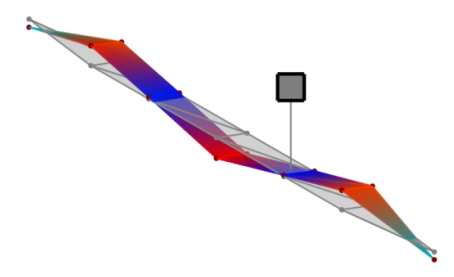

(d) Time : $t=29.6 \mathrm{~s}, f=98.11 \mathrm{~Hz}$

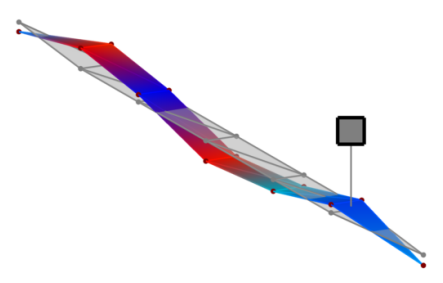

(e) Time : $t=37.4 \mathrm{~s}, f=84.96 \mathrm{~Hz}$

Figure 9: Time-varying shape of the sixth mode at particular time spots. The effect of the mass (represented by the gray square) is well visible in nodes/antinodes of resonances.

In our case, there is no kinematic constraint to fulfil and the vertical displacement and rotation fields are expressed in a polynomial basis :

$$
\left\{\begin{array}{r}
z(x)=\sum_{i=0}^{n} w_{i}(x) q_{z, i} \\
\theta(x)=\sum_{i=0}^{n} w_{i}(x) q_{\theta, i}
\end{array}\right.
$$

with

$$
w_{i}(x)=\left(\frac{\left(x-\frac{L}{2}\right)}{L}\right)^{i} .
$$

Polynomial functions up to the power $i=10$ are sufficient to have converged results for the following position-varying analysis.

In this model, the bending and torsion modes are decoupled in two problems that are solved separately and the results are merged at the end. The results can be compared with those obtained previously in the LTI modal analysis in Section 2.2 and the correlation between the experimental results and the Rayleigh-Ritz model is summarized in the form of a Modal Assurance Criteria (MAC) matrix shown in Figure 11.

\subsection{Introduction of the moving mass}

Now that the beam subsystem is modeled, the moving mass can be introduced in the inertia part of the problem. The moving mass will punctually act on the kinetic energy at its instantaneous location. The additional contributions considered here are the the mass in vertical translation and the inertia of the steel block in rotation about the $y$ - and $x$-axes for the bending and torsion deformations, respectively. 


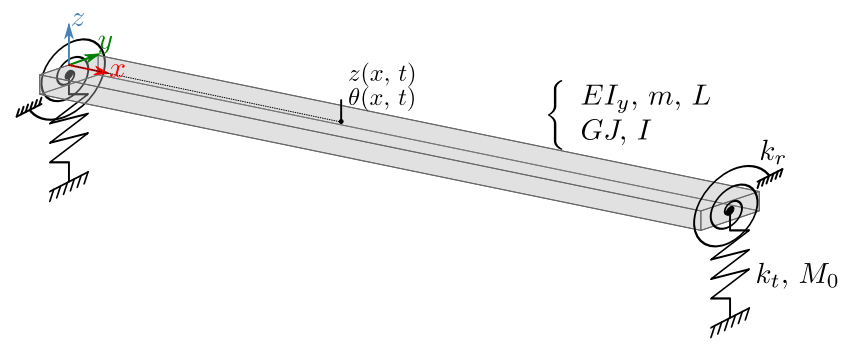

Figure 10: Model of the beam for the Rayleigh-Ritz approach.

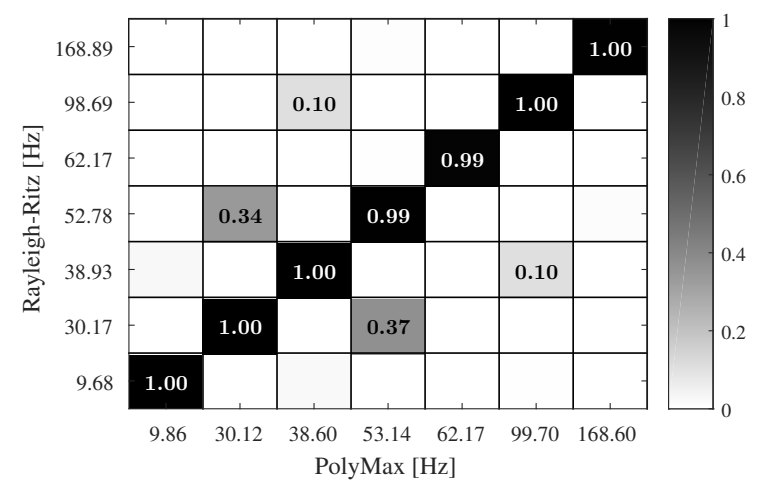

Figure 11: Correlation matrix between the mathematical model of the LTI subsystem.

To compare the results from the Rayleigh-Ritz model with those of the time-varying identification, 50 equally spaced time steps are selected in the time span under study and the related position of the mass for these time steps are interpolated from the recorded location data in Figure 4.

The solution from the mathematical model in its 50 samples is plotted in Figure 12 together with the time-varying identification. Two remarks can be drawn from that plot. First, the mathematical model obtained by simply introducing the moving mass in the initial updated model matches globally well the identified results. The largest discrepancies between the two sets of results lie at the beginning and at the end of the last time-varying mode. Note that the divergence at the end is related to the fast change in the location data close to 40 seconds.

Finally, having compared the frequencies, the mode shapes can be analyzed too. Traditionally, the mode shape correlation is presented in a 2D MAC matrix representing the correlation between the modes of one set with all the other modes of the other set. Here, because the modes are no more constant, the traditional layout of the MAC matrix is modified to integrate the time dimension. For each time instant of the Rayleigh-Ritz model, the MAC matrix coefficients are calculated then stacked in a column form. All the instantaneous correlations are then put one after each other to create a time-varying MAC matrix as shown in Figure 13. This kind of plot may be not optimal because it rapidly becomes cumbersome as the number of modes increases, it has the advantage to make the correlation lines visible. For most of the modes, the correlation between the Rayleigh-Ritz model and the experimental results are pretty good all along the time line. Only the fifth experimental mode is concerned by a weaker correlation with its mathematical counterpart. The reason lies in the fact that experimental modes 4 and 5 are very close and cross themselves several times during the recording process. The third mode is also concerned by a lack of correlation but only during the first second. 


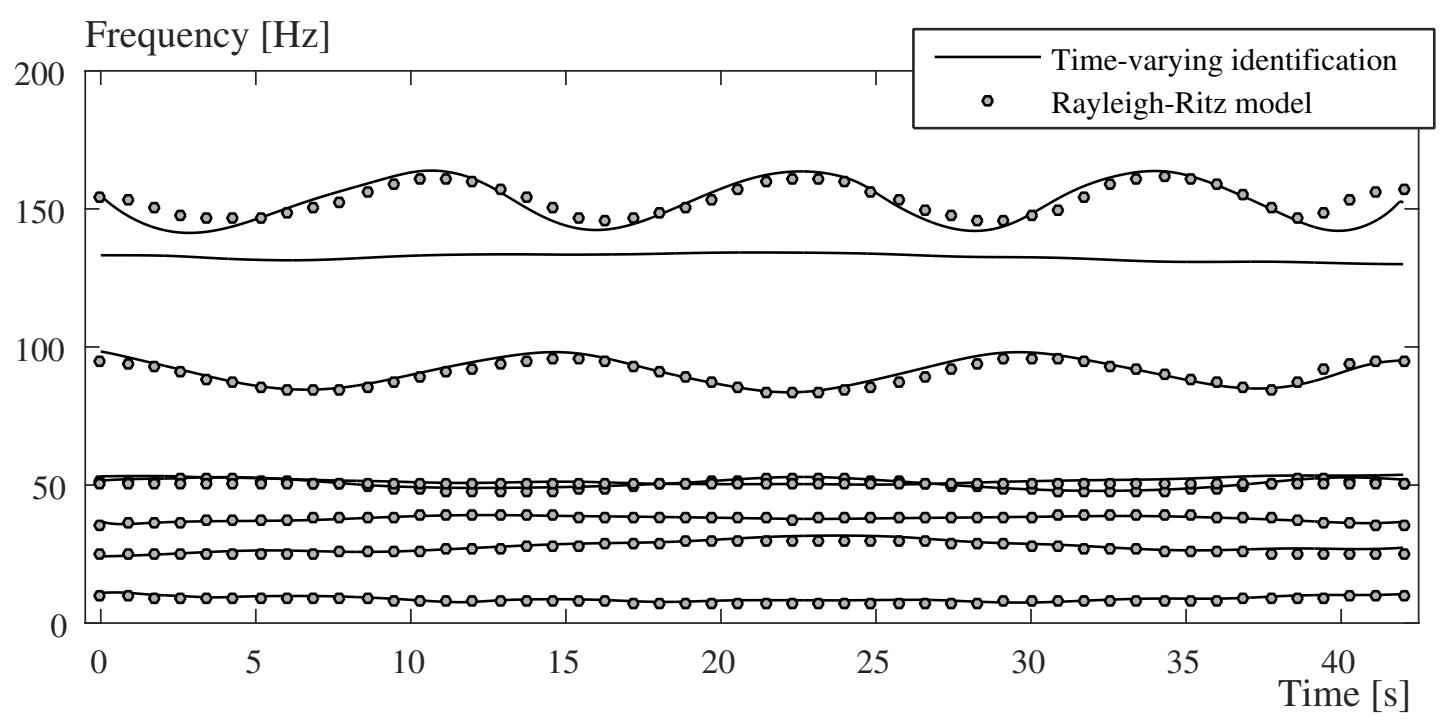

Figure 12: Comparison of the time-varying identification and the Rayleigh-Ritz model.

\section{Use of the instantaneous mode shapes to track the variation}

The instantaneous mode shapes of the system may be used to track structural changes. To this end, tools initially developed for damage localization can be used. Model updating methods can be used to detect and locate errors or damages between numerical and experimental data [33] but they usually require the knowledge of the structural matrices obtained from a finite element model of the structure. In this paper, we choose to use the Coordinate Modal Assurance Criterion (COMAC) [34] or its enhanced version (eCOMAC) [35] which are tools able to correlate the coordinates between two sets of modes. In the following, we compare the set of experimental data of the time invariant system (beam and supports only) presented in Section 2.2 and the results of the corresponding Rayleigh-Ritz model presented in Section 4.1.

The eCOMAC criterion is a vector containing as many coefficients as the number of degrees-of-freedom contained in the mode shapes. It is computed as follows:

$$
e C O M A C(i)=\frac{\sum_{j=1}^{N_{m}}\left|\boldsymbol{X}_{j}(i)-\boldsymbol{Z}_{j}(i)\right|}{2 N_{m}}
$$

in which $\boldsymbol{X}$ and $\boldsymbol{Z}$ are two matrices containing two sets of corresponding mode shapes (for example from numerical and experimental analyses) and $N_{m}$ is the number of modes used for the correlation. Let us note that for the eCOMAC criterion, the two sets of modes have to be unit-normalized and the modes in each pair have to be in phase.

\subsection{Mass tracking using only experimental results}

Having performed a linear time invariant modal analysis of the system before the introduction of the moving mass, its results can be used as a reference for the tracking of the structural modification due to the presence of the mass. In Figure 14 the sixth mode of the beam in its nonperturbed condition (Figure 14(a)), the same mode in the system containing the moving mass identified at $t=6.7 \mathrm{~s}$, (Figure 14(b)) such as in Figure 9(a) and the eCOMAC values distributed on the experimental mesh are reported. The sixth mode is used here because of the visual effect of the mass for that mode at that particular time instant but all the modes are obviously retained for the criterion calculation.

The eCOMAC criterion is calculated for each time step. Because of the rather rough experimental mesh, a cubic interpolation of the criterion is used between successive nodes along the beam to increase the spatial resolution. The position of its maximum value gives us our approximate identified position of the mass. 


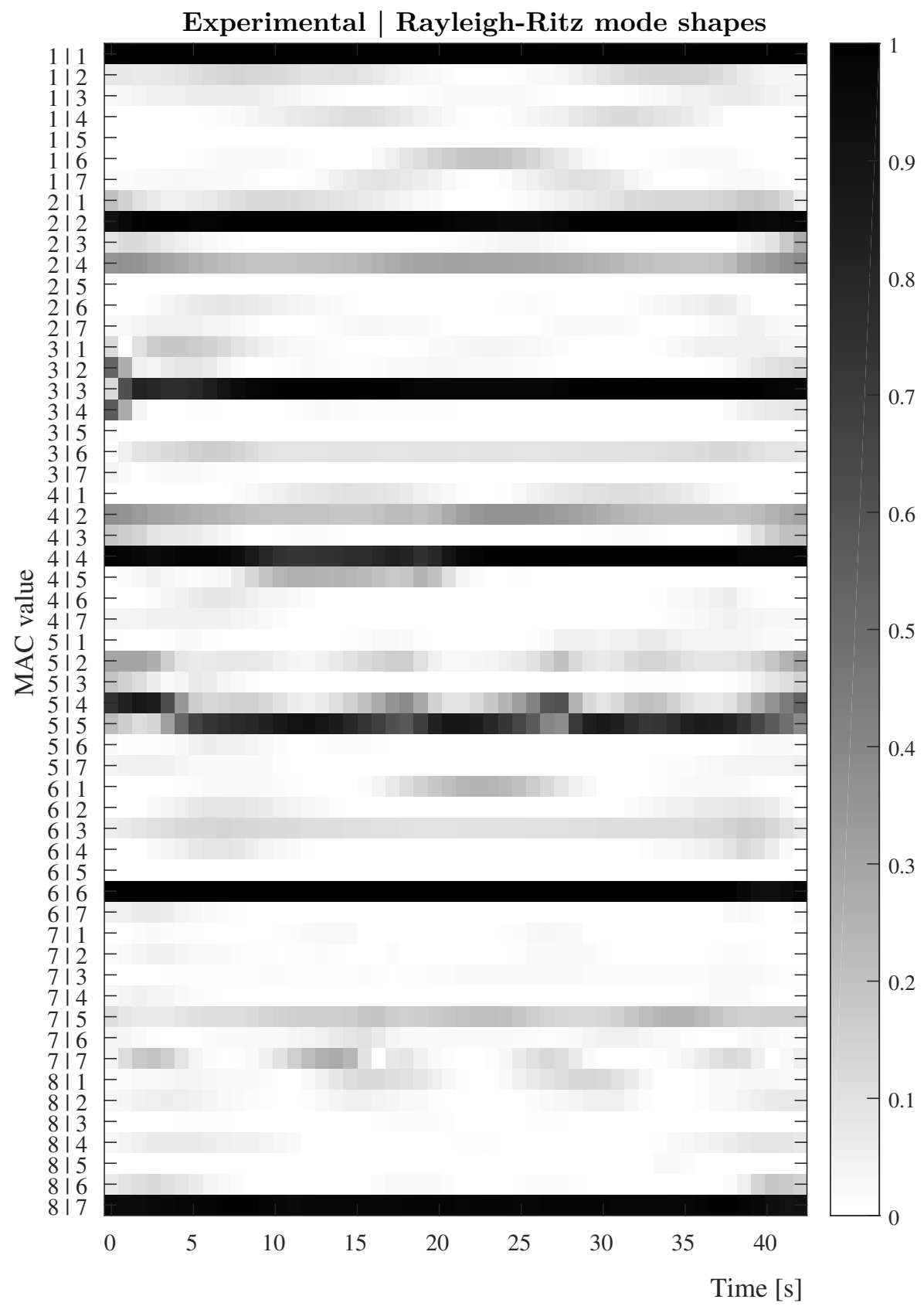

Figure 13: Time-varying mode shape correlation. 


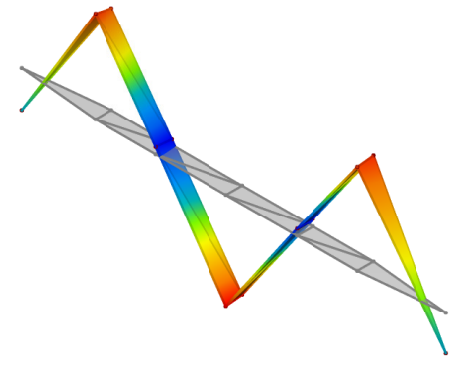

(a) Sixth LTI mode shape.

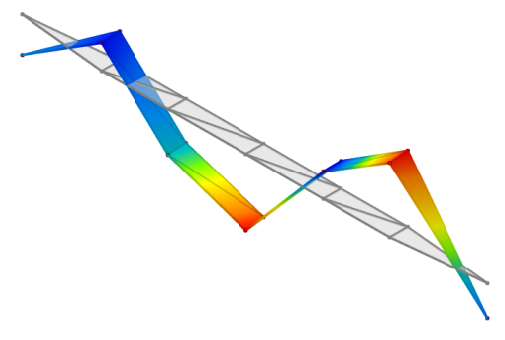

(b) Sixth TV mode shape at $t=6.7 \mathrm{~s}$.

(c) eCOMAC distribution at $t=6.7 \mathrm{~s}$.

Figure 14: Comparison between the reference experimental set of modes and the time-varying identified ones with the eCOMAC criterion.

This is represented in Figure 15 by white dots. In this figure, the black dots represent the actual (measured) position of the center of the mass and the surrounded grey area represents a $\pm 5 \mathrm{~cm}$ curve to represent the length of the steel block. We can see that the global motion is caught but the discrepancies between the measured and estimate position may be large at some instants.

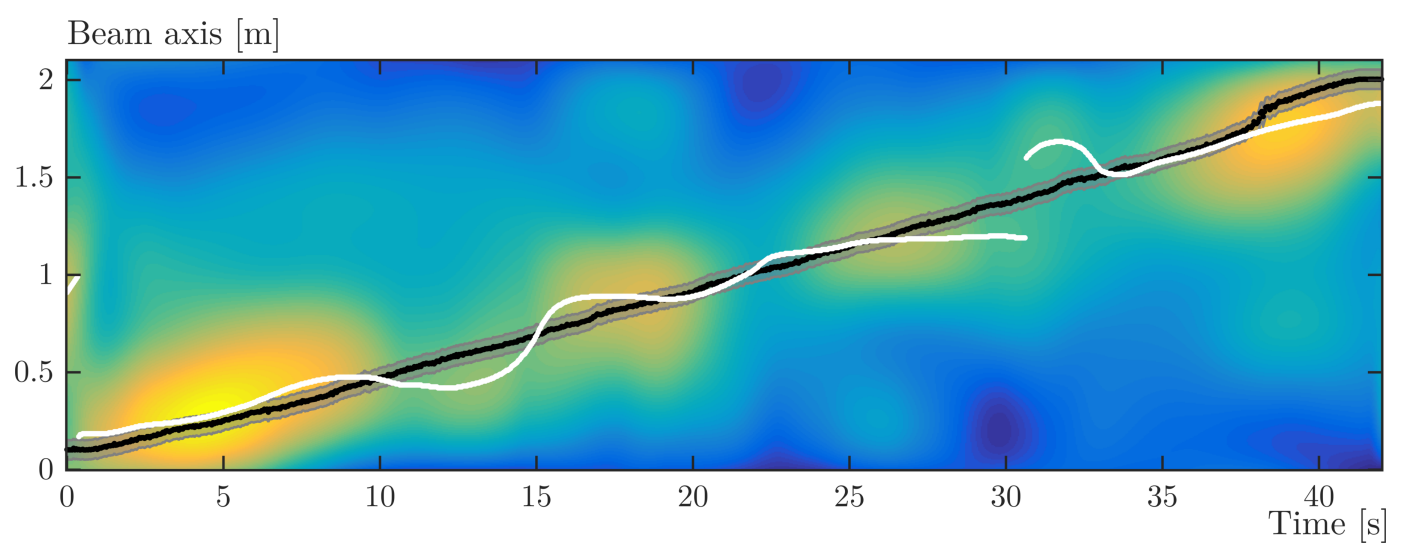

Figure 15: Estimated position of the mass by the eCOMAC criterion. The black and white dots represent the measured and estimated position of the mass, respectively. 


\subsection{Mass tracking using experimental results and the first mathematical model}

A second approach to track the modification of the system with time is to use our first Rayleigh-Ritz model. As shown previously in Section 4.1, this model well represents the structure. The advantage of the results from that model instead of the results of the LTI modal analysis is that the model is a continuous approximation of the mode shapes. It follows that we can spatially discretize it at any coordinate about the beam axis and then refined results may be obtained far easily. The problem is then how to compare our rough measurement mesh with the refined numerical one. To do so, we use here expansion methods to expand our measurements results to the refined numerical mesh. The System Equivalent Reduction Expansion Process (SEREP) [36] method is used to expand our results. The aim of the method is to use a set of analytical modes to expand the experimental ones. First, let us separate the analytical degrees-of-freedom into two subsets, the $m$ masters corresponding to the measured DoFs, and the $s$ slaves DoFs. If $\boldsymbol{Z}$ represents the set of experimental mode shapes and

$$
\boldsymbol{X}=\left[\begin{array}{c}
\boldsymbol{X}_{m} \\
-\overline{\boldsymbol{X}}_{s}^{-}
\end{array}\right]
$$

the (smoothed) expanded set of experimental modes is given by

$$
\boldsymbol{Z}_{\text {expanded }}=\left[\begin{array}{c}
\boldsymbol{Z}_{m} \\
-\boldsymbol{Z}_{s}^{-}
\end{array}\right]=\left[\begin{array}{c}
\boldsymbol{X}_{m} \\
-\overline{\boldsymbol{X}}_{s}^{-} \\
\overline{\boldsymbol{T}}^{-}
\end{array}\right]
$$

in which the transformation matrix $\boldsymbol{T}$ is computed with the experimental modes and the pseudoinverse $\left({ }^{\dagger}\right)$ of the analytical modes at the master nodes:

$$
\boldsymbol{T}=\boldsymbol{X}_{m}^{\dagger} \boldsymbol{Z}
$$

As in the previous section, Figure 16 represents the sixth refined initial mode shape (Figure 16(a)), the expanded sixth time-varying mode at $t=6.7 \mathrm{~s}$ (Figure 16(b)) ant the eCOMAC distribution on the refined mesh (Figure 16(c)).

Processing exactly in the same way as before, the results obtained using the refined mesh are closer to the actual position of the mass as shown in Figure 17. Regarding this result, the use of the expansion method increases the tracking precision of the position but it requires an analytical or numerical (finite elements model) modal basis, which is not always available.

\section{Conclusion}

In this paper, the problem of modal identification of linear time-varying systems was addressed. The present approach is based on the extension of the multivariate autoregressive moving average model using the method of the basis functions. A demonstration structure exhibiting a great time-dependence was used to validate the method on experimental data. The obtained results show the performance of the method to identify a set of time-varying modal parameters which are well representative of the physical behavior of the system. The results of the identification process were then compared with a mathematical model of the problem to ensure their validity. The drawback of the method is the number of computed spurious modes that may be large with respect to the number of physical modes. However the proposed selection method based on the mean phase deviation gives good results according to the example presented in the paper. Finally, the advantage brought by the use of a multivariate model is that the identification of the instantaneous mode shapes becomes possible. Some applications requiring the knowledge of the mode shapes are then possible and the example of the tracking of the variation of the system was shown. Because the position of the mass was recorded together with the response signals, it was possible to compare the estimated position with the actual one. 


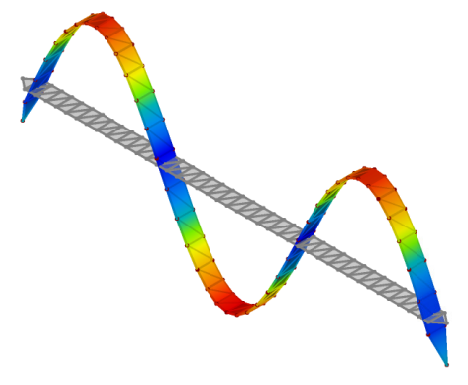

(a) Refined sixth LTI mode shape.

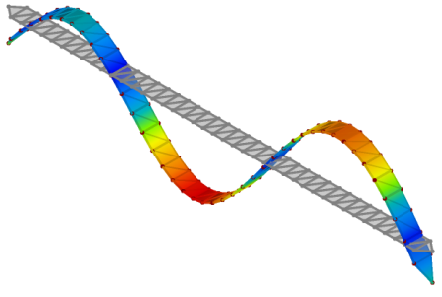

(b) Expanded sixth TV mode shape at $t=6.7 \mathrm{~s}$.

(c) eCOMAC distribution at $t=6.7 \mathrm{~s}$.

Figure 16: Finer localization using the mathematical model with a refined mesh. The expansion process of the experimental time-varying modes is illustrated to match the refined reference mesh.

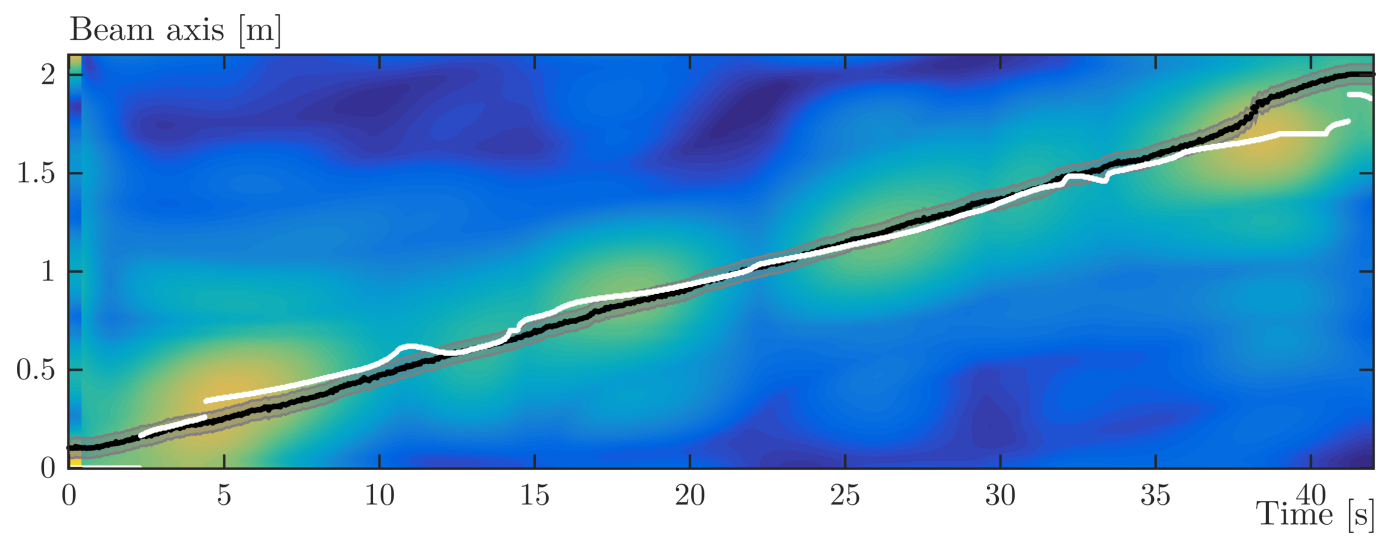

Figure 17: Estimated position of the mass by the eCOMAC criterion with the refined mesh. The black and white dots represent the measured and estimated position of the mass, respectively. 


\section{References}

[1] L. Garibaldi, S. Fassois, MSSP special issue on the identification of time varying structures and systems, Mechanical Systems and Signal Processing 47 (1-2) (2014) 1-2.

[2] S. Marchesiello, S. Bedaoui, L. Garibaldi, P. Argoul, Time-dependent identification of a bridge-like structure with crossing loads, Mechanical Systems and Signal Processing 23 (6) (2009) 2019-2028.

[3] M. Goursat, M. Döhler, L. Mevel, P. Andersen, Crystal Clear SSI for Operational Modal Analysis of Aerospace Vehicles, in: Proceedings of the IMAC-XXVIII, 2010, pp. 1421-1430.

[4] B. Basu, S. Nagarajaiah, A. Chakraborty, Online Identification of Linear Time-varying Stiffness of Structural Systems by Wavelet Analysis, Structural Health Monitoring 7 (2008) 21-36.

[5] X. Xu, Z. Shi, Q. You, Identification of linear time-varying systems using a wavelet-based state-space method, Mechanical Systems and Signal Processing 26 (2012) 91-103.

[6] W. J. Staszewski, D. M. Wallace, Wavelet-based Frequency Response Function for time-variant systems - An exploratory study, Mechanical Systems and Signal Processing 47 (1-2) (2014) 35-49.

[7] N. E. Huang, S. S. Shen, Hilbert-Huang Transform and its Applications, Vol. 5 of Interdisciplinary Mathematical Sciences, World Scientific Publishing Co. Pte. Ltd., 2005.

[8] M. Feldman, Hilbert Transform Applications in Mechanical Vibration, John Wiley \& Sons, Ltd, Chichester, UK, 2011.

[9] Z. Shi, S. Law, X. Xu, Identification of linear time-varying MDOF dynamic systems from forced excitation using Hilbert transform and EMD method, Journal of Sound and Vibration 321 (3-5) (2009) 572-589.

[10] M. Bertha, J.-C. Golinval, Experimental modal analysis of a beam travelled by a moving mass using Hilbert Vibration Decomposition, in: Proceedings of Eurodyn IX, Porto, 2014.

[11] M. Bertha, J.-C. Golinval, Modal identification of time-varying systems using Hilbert transform and signal decomposition, in: Proceedings of the International Conference on Noise and Vibration Engineering, ISMA 2014, Leuven, 2014, pp. 2409-2419.

[12] K. Liu, Identification of Linear Time-Varying Systems, Journal of Sound and Vibration 206 (4) (1997) 487-505.

[13] K. Liu, Extension of modal analysis to linear time-varying systems, Journal of Sound and Vibration 226 (1) (1999) $149-167$.

[14] K. Liu, L. Deng, Identification of pseudo-natural frequencies of an axially moving cantilever beam using a subspace-based algorithm, Mechanical Systems and Signal Processing 20 (1) (2006) 94-113.

[15] Y. Grenier, Time-dependent ARMA modeling of nonstationary signals, IEEE Transactions on Acoustics, Speech, and Signal Processing 31 (4) (1983) 899-911.

[16] Y. Grenier, Modèles ARMA à coefficients dépendant du temps estimateurs et applications, Traitement du signal 3 (1986) 219-233.

[17] T. S. Rao, The Fitting of Non-stationary Time-Series Models with Time-Dependent Parameters, Journal of the Royal Statistical Society. Series B (Statistical Methodology) 32 (2) (1970) 312-322.

[18] M. Spiridonakos, A. Poulimenos, S. Fassois, Output-only identification and dynamic analysis of time-varying mechanical structures under random excitation: A comparative assessment of parametric methods, Journal of Sound and Vibration 329 (7) (2010) 768-785.

[19] M. Spiridonakos, S. Fassois, Non-stationary random vibration modelling and analysis via functional series time-dependent ARMA (FS-TARMA) models - A critical survey, Mechanical Systems and Signal Processing 47 (1-2) (2013) 1-50.

[20] M. D. Spiridonakos, S. D. Fassois, Parametric identification of a time-varying structure based on vector vibration response measurements, Mechanical Systems and Signal Processing 23 (6) (2009) 2029-2048.

[21] P. Andersen, Identification of civil engineering structures using vector ARMA models, Ph.D. thesis (1997).

[22] J.-B. Bodeux, J.-C. Golinval, Application of ARMAV models to the identification and damage detection of mechanical and civil engineering structures, Smart Materials and Structures 10 (3) (2001) 479-489.

[23] B. Piombo, E. Giorcelli, L. Garibaldi, A. Fasana, Structures Identification Using ARMAV Models, in: Society for Experimental Mechanics (Ed.), IMAC 11, International Modal Analysis Conference, Orlando, 1993, pp. 588-592.

[24] P. Andersen, R. Brincker, P. H. Kirkegaard, Theory of covariance equivalent ARMAV models of civil engineering structures, in: IMAC XIV - 14th International Modal Analysis Conference, Vol. 71, 1995.

[25] L. Ljung, System Identification : Theory for the User, 1999.

[26] L. A. Zadeh, Frequency analysis of variable networks, Proceedings of the IRE 27 (3) (1950) 170-177.

[27] K. Zenger, R. Ylinen, Poles and Zeros of Multivariable Lienar Time-Varying Systems, Proc. of the 15th IFAC World Congress.

[28] S.-D. Zhou, W. Heylen, P. Sas, L. Liu, Parametric modal identification of time-varying structures and the validation approach of modal parameters, Mechanical Systems and Signal Processing 47 (1-2) (2014) 94-119.

[29] B. Peeters, H. Van der Auweraer, PolyMAX: A revolution in operational modal analysis, in: 1st International Operational Modal Analysis Conference, Copenhagen, 2005.

[30] M. Niedzwiecki, Identification of Time-Varying Processes, John Wiley \& Sons, Inc., New York, NY, USA, 2000.

[31] A. Beex, P. Shan, A time-varying Prony method for instantaneous frequency estimation at low SNR, in: ISCAS'99. Proceedings of the 1999 IEEE International Symposium on Circuits and Systems VLSI (Cat. No.99CH36349), Vol. 3, 1999, pp. 5-8.

[32] D. Rixen, M. Geradin, Mechanical vibrations: theory and application to structural dynamics, 2nd Edition, John Wiley \& Sons, Ltd, 1997.

[33] M. Friswell, J. E. Mottershead, Finite Element Model Updating in Structural Dynamics, Springer, 1995.

[34] N. A. J. Lieven, D. J. Ewins, Spatial correlation of mode shapes: the coordinate modal assurance criterion (COMAC), in: Proceedings of the 6th International Modal Analysis Conference (IMAC), 1988, pp. 690-695. 
[35] D. L. Hunt, Application of an enhanced Coordinate Modal Assurance Criterion, in: 10th International Modal Analysis Conference, San Diego, 1992, pp. 66-71.

[36] J. O'Callahan, P. Avitabile, R. Riemer, System Equivalent Reduction Expansion Process (SEREP), in: Seventh International Modal Analysis Conference, Las Vegas, 1989. 